\title{
Prácticas de Educación COMUNITARIA CRÍTICA DE LA UNIVERSIDAD \\ SURCOLOMBIANA EN EL MARCO DE LA EXTENSIÓN UNIVERSITARIA ${ }^{1}$
}

\author{
Critical Community Education Practices of \\ THE SURCOLOMBIANA UNIVERSITY WITHIN THE \\ FRAMEWORK OF THE UNIVERSITY EXTENSION
}

Nicolás Medina-Medina*

Recibido: Abril 27, 2021

Aceptado: Noviembre 4, 2021

* Magíster en Educación de la
Universidad Surcolombiana.
Enlace Regional de
Asistencia Técnica para la
Primera Infancia, Instituto
Colombiano de Bienestar
Familiar-Regional Huila.
nicolasmedina.20@gmail.
com
0000-0002-4708-0592

1 Prácticas de Educación Comunitaria Crítica de la Universidad Surcolombiana, 2021.

Cómo citar este artículo: Medina, M. N. (2021). Prácticas de Educación Comunitaria Crítica de la Universidad Surcolombiana en el marco de la Extensión Universitaria. Revista PACA 11, pp. 51-66.
Resumen: En el presente trabajo se realiza una aproximación interpretativa a las prácticas de educación comunitaria crítica de la extensión universitaria de la Universidad Surcolombiana, con base en los proyectos de Alfabetización del Pato Guayabero y el Macroproyecto DESCA; en el marco socio-histórico, contextualizado, situado y plural. Se reconocen las tensiones entre las posturas de la extensión universitaria y los contenidos de los proyectos en mención. $Y$, finalmente, se identifican seis prácticas de educación comunitaria crítica por sus saberes prácticos, sentires y materialidades.

Palabras clave: educación; educación comunitaria; prácticas de educación comunitaria; extensión universitaria; pedagogías críticas latinoamericanas.

Abstract: In the present work an interpretive approach is made to the critical community education practices of the university extension of the Surcolombiana University, based on the Guayabero Duck Literacy projects and the DESCA Macroproject; in the sociohistorical, contextualized, situated and plural framework. The tensions between the positions of the university extension and the contents of the projects in question are recognized. And, finally, six critical community education practices are identified for their practical knowledge, feelings and materialities. 


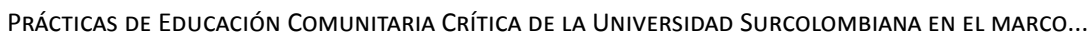

Keywords: community education; community education practices; college extension; latin american critical pedagogies.

\section{Introducción}

Las Prácticas de Educación Comunitaria Crítica de la Universidad Surcolombiana es una investigación que se desarrolla en el marco de la Maestría en Educación con Énfasis en Investigación y Docencia Universitaria, para realizar una aproximación interpretativa a los proyectos que se han desarrollado en Extensión Universitaria de la Surcolombiana.

En ese sentido, se describirán dos proyectos de investigación de extensión universitaria que se considera marcan la pauta y la actualidad de un modelo de universidad que se articula con el movimiento social y popular de la región. Y, a su vez, se mueven entre tensiones en un espaciotiempo en particular.

Los proyectos referenciados son: Alfabetización del Pato Guayabero que logró la formación en alfabetizados, postalfabetizados y alfabetizadores en la región. Por otro lado, se encuentra el Macroproyecto DESCA que fomentó el fortalecimiento organizativo a la Asociación de Afectados por el Proyecto Hidroeléctrico El Quimbo (Asoquimbo), conformado principalmente por campesinos del centro del departamento del Huila.

\section{Referentes Teóricos}

\section{Pedagogías críticas Latinoamericanas (PCL)}

Cuando se habla de las pedagogías críticas surten diversos elementos a considerar y reconocer; es decir, no es vista como una sola; en ese sentido y con base en Cabaluz-Ducasse (2016) se pueden conglomerar cuatro nodos generales que recogen las propuestas de Pedagogías Críticas Latinoamericanas:

1. Pedagogías contrahegemónicas: En el desarrollo teórico-práctico de las pedagogías críticas latinoamericanas, se ha venido constituyendo “(...) un cuerpo pedagógico negado, excluido y deslegitimado por las pedagogías oficiales (...) (como también aportan para crear) prácticas socioeducativas que cuestionen y transformen la sociedad existente" (p. 78). 
2. Pedagogías territorializadas: Desde esta perspectiva, no se desconoce el desarrollo epistemológico de paradigmas eurocentristas, pero sí se le apuesta a una construcción desde lo local, desde la razón instrumentalizada y desde el sentir, es decir, desde el sentipensamiento.

3. Pedagogías de la alteridad: Aquí se establece la importancia de un proyecto de vida que se forja como una opción ética-política por "(...) el/ la, otro/a excluido/a, explotado/a, dominado/a (...) en las circunstancias políticas, sociales y económicas de la alteridad, nos permite reconocer la concreción del oprimido (...) Solo conociendo y aproximándose a la realidad del Otro en cuanto Otro (...)" (p. 80).

4. Pedagogías de las praxis: Más allá de una construcción teórica, las Pedagogías Críticas Latinoamericanas buscan materializar sus planteamientos de transformación en las realidades cercanas a la comunidad, sin desconocer las perspectivas regionales, nacionales e internacionales.

\section{Educación Comunitaria (ECO)}

Las problematizaciones en la Educación Comunitaria tienen que ver con lo que se comprende por comunidad, comunitario y, el papel del educador y la educadora comunitaria en los territorios (Clavijo Ramírez, 2017). La comunidad es poder constituyente diverso y plural “(...) su naturaleza está tejida de múltiples fuerzas; fuerzas divergentes, sin duda, pero enfrentadas a su antagonismo natural porque rompen la cooperación social: el capitalismo" (Choachí González, 2017, p. 65).

Así, la comunidad como referente es un lugar epistémico donde hay expresiones individuales y colectivas que establecen proyectos comunes y propician nuevas subjetividades que cuestionan los quiebres principalmente del proyecto moderno implementado en los territorios (Cuevas Marín, 2017). En ese sentido, la unidad “(...) es un proceso de fragmentación permanente, mediado por la diferencia de intereses y de necesidades (...) Pese a este panorama, es evidente la emergencia de organización, en el sentido de hacer frente a problemáticas comunes que las transversalizan (...)" (Clavijo Ramírez, 2017, p. 35).

La educación comunitaria (ECO) reconoce las implicaciones de la relación saber-poder (Pérez Luna \& Sánchez Carreño, 2005), evidenciada en la producción de "(...) capital simbólico -saberes pedagógicos teóricos y prácticos- y de capital social -interacciones, profesiones- sobre la educación (...)" (Runge Peña, Gárces Gómez, \& Muñoz Gaviria, La Pedagogía como Campo Profesional y Disciplinar, p. 213), en consideración 
de las condiciones socio-históricas que posibilitan dichos capitales. En ese sentido, "No es sólo un debate en torno al replanteamiento de disciplinas y titulaciones, o de definición de competencias profesionales, sino también de comprometer territorial, institucional y socialmente la docencia y la investigación universitaria (...)" (Cierza García, 2006, p. 796).

\section{Prácticas de Educación Comunitaria}

Tener como referente la teoría de las prácticas es reconocer que estas son aprendidas y que no necesariamente deben ser conscientes. En ese sentido, no priorizan sobre el porqué sino sobre el saber cómo, el cómo se ejecuta una práctica en un determinado contexto, lo que las hace ser conjunto de esquemas de los comportamientos corporales que se presentan en "(...) cognoscitivos (saber) transubjetivos y adscripciones de sentido subjetivos rutinizados. En la medida en que esas prácticas no presuponen, en su carácter implícito, una intencionalidad, hay que investigarlas en su lógica realizativa (performativa) (...)" (Runge Peña, p. 2).

Ariztía (2017), retomando a Rekwitz y Shatzky, Shove et al. propone las prácticas como la interrelación entre espacio-tiempo a partir de los siguientes tres elementos: competencias, sentido y materialidades. La primera tiene que ver con saberes prácticos y habilidades que permiten la realización de una práctica. Segundo, los aspectos teleo-afectivos para establecer “(...) significado y necesidad de una práctica para quienes las ejecutan" (p. 225). Y finalmente, hace referencia a las herramientas, infraestructura y recursos para realizar la práctica; frente a este último punto es necesario notar que: "(.) las materialidades son constitutivas de las prácticas y no un elemento externo: definen la posibilidad de existencia de esta, así como sus transformaciones" (p. 225).

Desde Valladares (2017), retomando a Martínez y Huang (2015), la práctica educativa contiene una arquitectura en tres elementos para teorizar que son: local, historicista y pluralista, posicionando teóricamente el estudio como una perspectiva empírica, crítica e interpretativa. Así, lo local identifica "(...) las normas locales propias de prácticas educativas específicas y sus intercambios y flujos con otras prácticas, para configurar, así, proyectos educativos distinguibles (...)"; a su vez, historicista puesto que "(...) estas normas se sitúan, estabilizan y cambian en contextos históricos particulares $(. .$.$) "; y$, finalmente, pluralista debido a que en “(...) diferentes prácticas habrá distintos tipos de estándares para identificar aquello que se considera legítimo (de comprender y explicar o de contar como explicación o prescripción) dentro del campo educativo" (p. 202). 


\section{Metodología}

La presente investigación es de enfoque cualitativo, el cual sustenta una serie de prácticas que son materiales e interpretan el mundo para transformar; en ese sentido, se convierten en representaciones por medio de herramientas como notas de campo, entrevistas, conversaciones y notas para el investigador (Denzin \& Lincoln, 2012).

El diseño es con base en el estudio de casos. Es importante mencionar que el estudio de casos para este proyecto trasciende, para posicionarse como un diseño que se fundamenta en la comparación de hallazgos y resultados entre los casos estudiados. "De esta manera, a partir de la comparación de un número limitado de casos seleccionados en función del propósito de la investigación, se replican los hallazgos y resultados de la misma" (Vasilachis de Gialdino, 2006, p. 225).
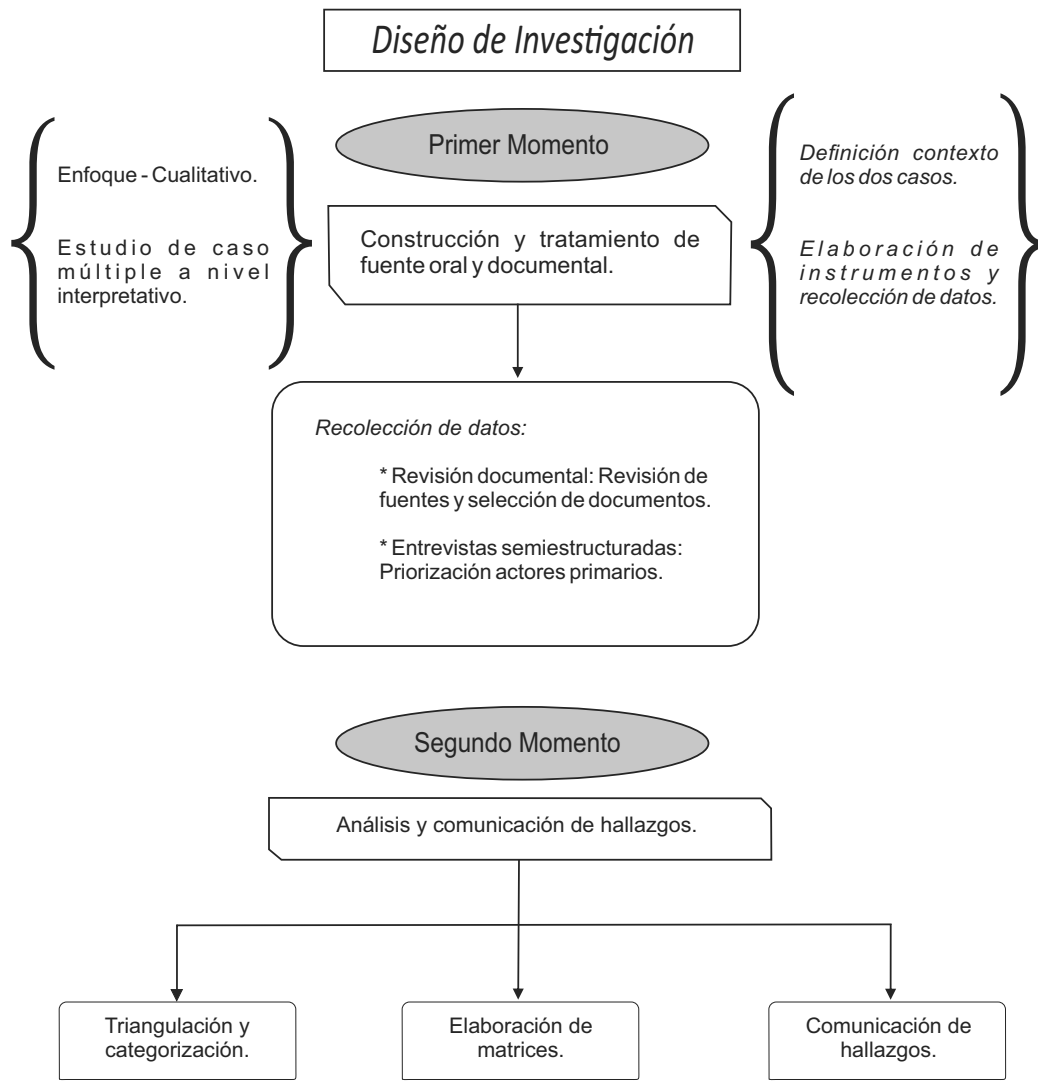

Figura 1. Diseño Investigación Prácticas de Educación Comunitaria Crítica de la Universidad Surcolombiana.

Fuente: Elaboración propia. 
De esta manera, se hace tratamiento de fuentes orales y escritas para la contextualización de los casos, elaboración de instrumentos y recolección de la información a partir de las técnicas de revisión documental y entrevista semiestructurada. Seguidamente, se analiza categóricamente la información (oral y escrita) desde lo propuesto en las prácticas de educación comunitaria.

Las categorías desde donde se indagan los proyectos objeto de estudio son: Saber práctico; Sentires y Materialidades. En la revisión documental se identifican: tiempo, objetivo, contexto, población, metodología, impactos y reflexiones; aplica, en el caso del proyecto de Alfabetización, texto inédito: En el Pato aprendimos a ser maestros, de Luis Ignacia Murcia Molina. Para el DESCA se cuenta con proyectos e informes presentados ante proyección social de la Universidad Surcolombiana. Y, en las entrevistas semiestructuradas, se realizan a docentes que han participado en los proyectos.

Se hace uso del estudio de caso de aproximación interpretativa porque los casos permiten discutir presupuestos teóricos relacionados con las prácticas de educación comunitaria. De igual forma, se realiza desde la modalidad de sistematización de experiencias, la cual se centra "(...) en reconstruir una experiencia que el grupo o el investigador consideran como significativa, y que supone la participación del grupo en su desarrollo" (Galeano Marín, 2012, p. 72).

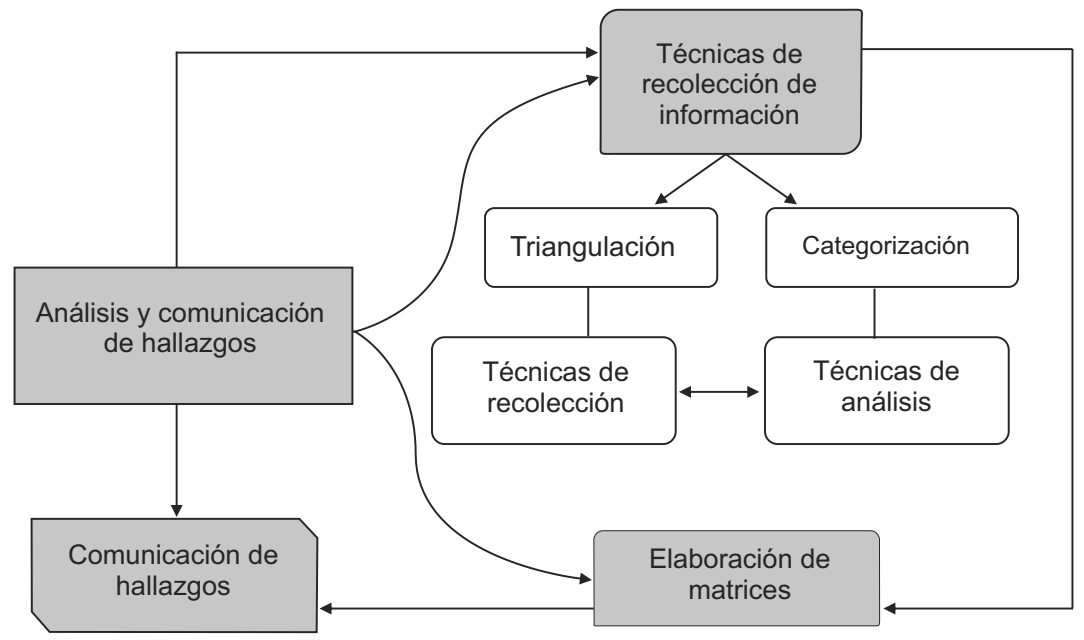

Figura 2. Análisis de la Investigación Prácticas de Educación Comunitaria Crítica de la Universidad Surcolombiana.

Fuente: Elaboración propia. 


\section{Resultados}

A partir de las pedagogías críticas latinoamericanas desde la perspectiva de Cabaluz-Ducasse (2016) y sus nodos centrales, se reconocen elementos político-pedagógicos. Asimismo, los aportes desde la educación comunitaria en lo ético-metodológico; y la base para comprender las prácticas de educación comunitaria desde los saberes prácticos, sentires y materialidades, permiten construir las categorías de análisis para identificar el desarrollo de las prácticas de educación comunitaria crítica en la extensión de la Universidad Surcolombiana con el estudio de estos dos casos.

En la extensión de la Universidad Surcolombiana desde el año de 1968 hasta el año 2010 se identifican las tensiones sobre políticas educativas y extensión universitaria en lo local, nacional e internacional. En esa medida, se distribuye en tres momentos: primer momento, que se encuentra entre 1968 y 1983, se denomina La universidad nació en la calle: materialización de un sueño colectivo; segundo momento, entre 1984 y 2001, La universidad del pueblo: democratización de la educación; y un tercer momento, entre 2002 y 2018, La universidad acreditada: materialización de la política educativa neoliberal.

Se establecen los momentos según los desarrollos de extensión de la Universidad Surcolombiana. A su vez, se tuvo presente la propuesta de temporalidad histórica que desarrolla Baicué Peña (2009) en el trabajo denominado Educación superior un espacio de frontera. Historia y desarrollo académico de la Universidad Surcolombiana, 1970-2010; esta propuesta se distribuye en cinco momentos históricos donde se reconstruye la historia de la Universidad: primer momento 1970-1975; segundo momento 1976-1983; tercer momento 1984-1990; cuarto momento 1991-1999; y quinto momento 2000-2010.

Es necesario reconocer que la Universidad Surcolombiana ha transitado por tres grandes bloques de extensión universitaria: primero, una perspectiva paternalista de garantizar la oferta académica; segundo, una apuesta transformadora de construcción con las comunidades, heredera de la exigencia de los movimientos sociales y populares; y, tercero, una institución instrumentalizada e instrumentalizadora que responden a las políticas neoliberales. 
Prácticas de Educación ComunitaRia Crítica de la Universidad SURColombiana en el Marco...

A continuación, se presenta la síntesis de los resultados que arrojó la investigación:

Tabla 1

Síntesis estudio de casos.
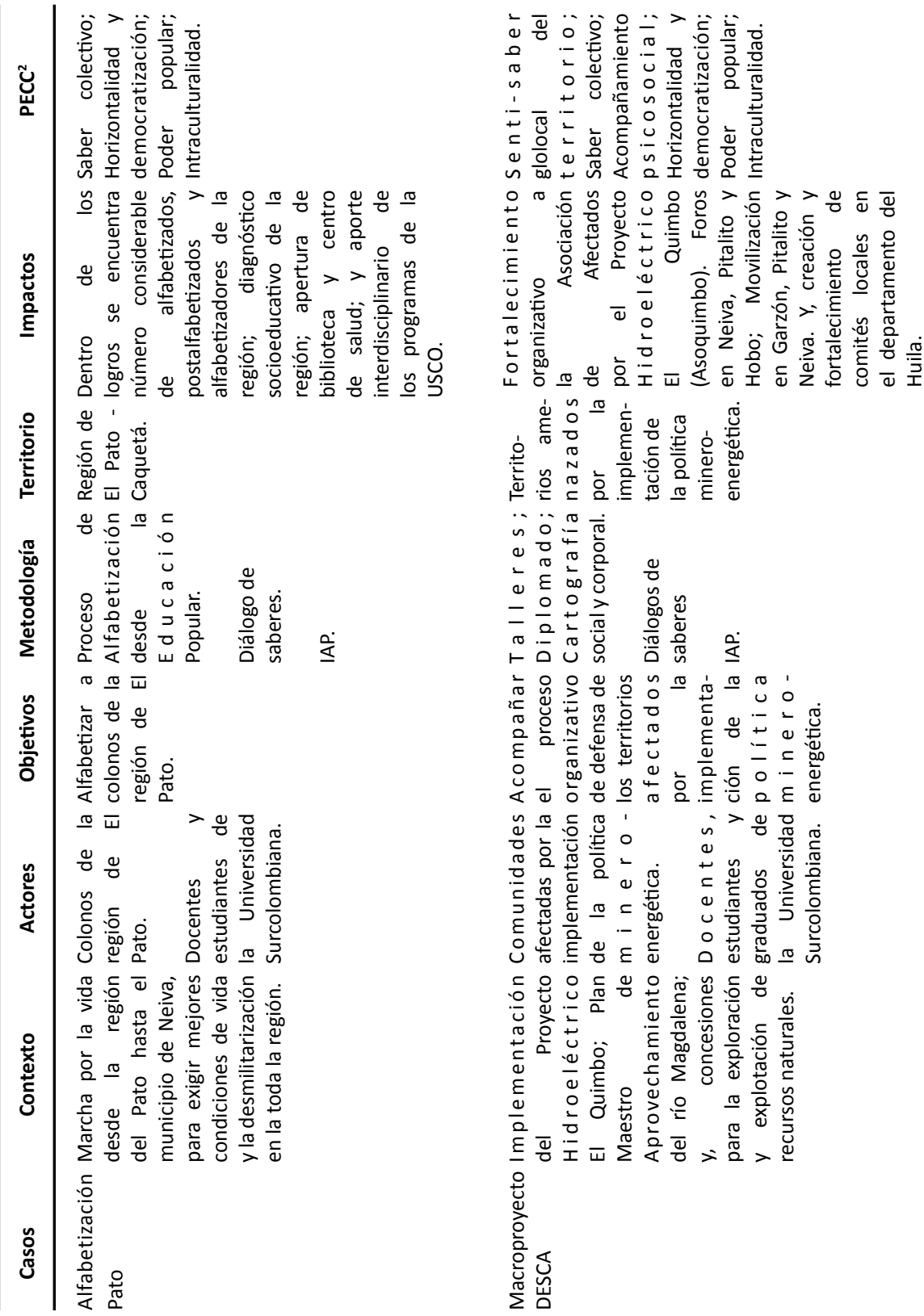

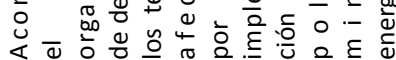
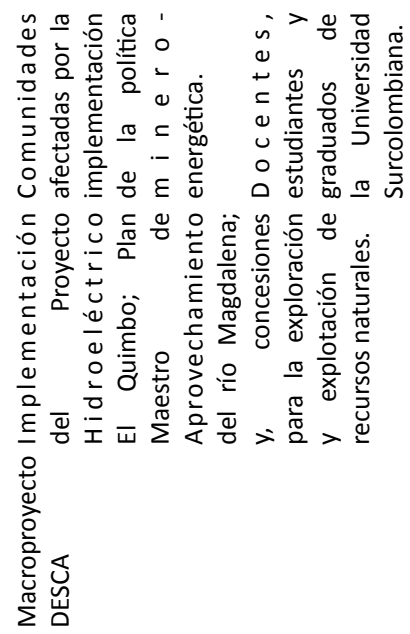

2 Prácticas de Educación Comunitaria Crítica.

Fuente: Elaboración propia. 


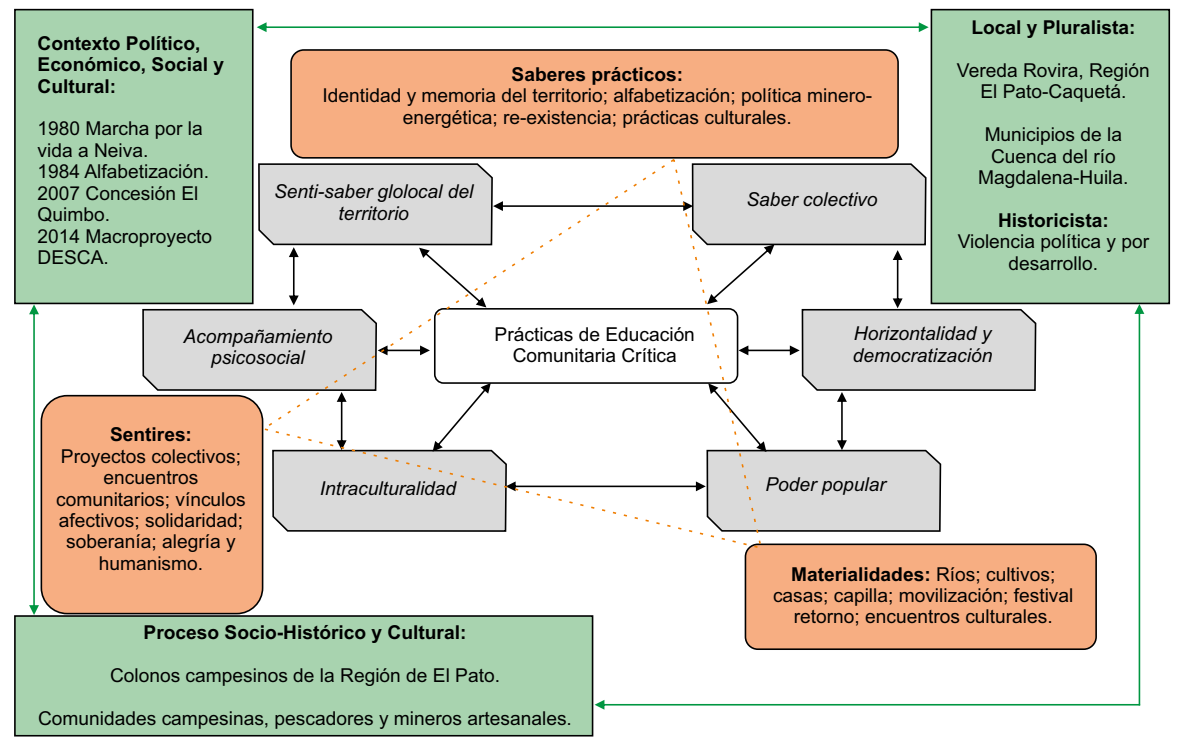

Figura 3. Prácticas de Educación Comunitaria Crítica Casos Alfabetización de El Pato Guayabero y Macroproyecto DESCA.

Fuente: Elaboración propia.

\section{Discusión}

A continuación, se exponen las prácticas de educación comunitaria crítica identificadas en el análisis:

\section{Senti-saber glolocal ${ }^{3}$ del territorio}

El Senti-saber glolocal del territorio hace alusión fundamentalmente a la realidad cotidiana en los territorios y surge a partir del reconocimiento del territorio y la reconstrucción de la vida comunitaria para visibilizar las prácticas que se afectaron a partir de la implementación de la política minero-energética en el departamento del Huila pero, a su vez, para proyectar el sentir colectivo de las comunidades sobre sus territorios.

3 El concepto Glolocal implica la interacción y punto de encuentro entre lo global y lo local. Para ampliar el concepto se sugiere revisar el texto de Arturo Escobar denominado El lugar de la naturaleza y la naturaleza del lugar: ¿globalización o postdesarrollo? 
Por las acciones desarrolladas en esta práctica se evidencian las formas de leer el territorio desde la perspectiva de las comunidades y, por tanto, se hicieron aproximaciones al territorio. En los mapas desarrollados por cartografía social y corporal queda plasmada la percepción que las comunidades tienen de su entorno y la dinámica que se vive en territorio con sus propias características.

No es una representación universal, sino que corresponde a un espaciotiempo en particular acotado y localizado que hace de ese territorio un lugar glolocalizado por la tensión que genera entre la implementación del proyecto hidroeléctrico a manos de una empresa transnacional y las comunidades que tienen otros deseos. En ese sentido, se “(...) vincula con los aspectos éticos, morales, axiológicos y políticos del hacer cotidiano de quienes participan (...)" (Barragán Girlado, 2016, p. 253).

\section{Saber colectivo}

El saber colectivo se construye a partir de saberes y experiencias que tienen las personas que confluyen en el proyecto. Así, la tarea del educador "(...) no es colocarse como sujeto cognocente, frente a un objeto cognocible para, después de conocerlo, hablar sobre él discursivamente a sus educandos, cuyo papel sería el de archivadores de sus comunicados" (Freire, 1984, p. 77).

Se construye saber "(...) ecologizado e integrador, que responde a una lógica dialógica, de complementariedad con todos y todas, en cualquier lugar y en cualquier momento" (Valdés Gutiérrez, 2018, p. 322). Surge una relación “(...) a partir del vínculo que los constituye. Sólo en esa medida, el diálogo de saberes puede ser realmente problematizador: hay un sujeto que confronta, cuestiona y por tanto transforma y es transformado" (Acevedo, Gómez, \& Zúñiga, 2016, p. 19).

\section{Acompañamiento Psicosocial}

Se acompaña reconociendo los diferentes ámbitos de existencia en los que se encuentran vinculadas las personas de la comunidad: Trabajo, Lúdico, Naturaleza, Autoridad colectiva, Inter/Subjetividad y, EróticoAfectivo. En cada ámbito, se vieron rupturas en los grupos poblacionales que fue necesario acompañar para identificar, contener y sanar. 
En el acompañamiento las personas se asumen en las relaciones que establecen con la comunidad y el territorio; es comprender a las personas que han sido sistemáticamente explotadas, excluidas e individualizadas para romper apuestas colectivas solidarias y generar un control territorial e implementar la política minero-energética en la lógica de desarrollo.

\section{Horizontalidad y Democratización}

La horizontalidad es una co-labor donde se consensuan acuerdos con base en las necesidades de cada persona que está actuando en el proceso. En ese sentido, “(...) comprometerse no solo puede ser interpretado de diversos modos, sino que involucra prácticas que (...) cuando las pensamos y articulamos en conjunto que se podrían abrir formas otras de producción de conocimiento, en términos metodológicos, teóricos, epistemológicos, políticos y éticos" (Briones, 2020, pp. 66-67).

Por otro lado, la democratización es la materialización de ser participe en los procesos formativos a quienes no tienen el privilegio de ingresar a la universidad. Siendo la educación un bien común, les pertenece a las comunidades y no necesariamente debe estar mediada por una institución educativa; sin embargo, al considerarse un derecho debe ser garantizada por el Estado; por ende, es una responsabilidad de las instituciones públicas garantizarla.

Hay que trascender de las formas de producción del conocimiento jerárquico y producir conocimiento desde la horizontalidad y democratización, hay que transgredir la "(...) "bio-lógica", donde las categorías binarias de género y raza, y su lectura fenotípica, jerarquizan asimismo la producción de conocimiento y definen quiénes son las autoridades científicas legitimadas para habitar las universidades y los hospitales, sus recursos y sus tecnologías" (Nogueira Beltrão, 2020, p. 236).

\section{Poder Popular}

En relación con esta práctica de educación comunitaria crítica se retomarán sólo tres de esos seis ámbitos que menciona Mejía (2016): El primero, es el Ámbito de vinculación a lo público donde hace referencia a que los intereses colectivos de actores inmersos en el proyecto se lleven al escenario público para ser disputado porque está en construcción. 
Seguidamente, se encuentra el Ámbito de vinculación a movimientos y formas organizadas inherente a la idea de construcción colectiva porque desde la organización se dan forma y fortalecen los intereses colectivos desde la realidad de cada comunidad y territorio. Es decir, es la "(...) construcción de otros mundos posibles" (p. 241).

El tercer ámbito es el Ámbito de construcción de procesos de gobernabilidad, que con base en las prácticas de educación comunitaria crítica descritas, es de ubicar mejor como Soberanía y no de Gobernabilidad, porque es la Soberanía de los pueblos la que está en proceso de construcción al reconocer el “(...) poder de los grupos populares (que) se construye y alcanza tras recorrer múltiples caminos (...)” (p. 241).

\section{Intraculturalidad}

Intraculturalidad se expresa por medio de la alegría y la festividad en relación con las prácticas y particularidades sociohistóricas en los territorios, es decir, que está sujeta a las comunidades que de generación en generación se han establecido en determinado lugar, sin desconocer los procesos socioculturales de globalización.

En esa medida, "Setrata deir más allá, detrascenderla lógica antihumanista de la mercantilización de la vida, del trabajo, de la naturaleza, del amor, del arte, del compañerismo, del sexo, que impulsa la transnacionalización irrefrenable del capital" (Valdés Gutiérrez, 2018, p. 343).

Hay que acabar con las prácticas que niegan la intraculturalidad y pluridiversidad donde se niega al otro “(...) a nombre de lo universal, los saberes a nombre de la ciencia, lo comunitario a nombre del individuo y lo personal, lo multicultural a nombre de lo monocultural, los sentidos a nombre de la razón (...)" (Mejía, 2016, p. 246).

\section{Conclusiones}

Se logra identificar el desarrollo de las prácticas de educación comunitaria crítica en la Universidad Surcolombiana desde los dos casos seleccionados de extensión universitaria, evidenciando los aportes que se vienen gestando desde la movilización social y popular para que el proyecto de Alfabetización se implemente en la región de El Pato. 
Por otro lado, el macroproyecto DESCA demuestra la tensión que se vive al interior de la Universidad Surcolombiana frente a dos modelos que están en disputa. De igual forma, recoge la tradición que inicia con el proceso de Alfabetización y que va a ser constante hasta aproximadamente el 2001. En los años siguientes, no va a ser visible hasta la aparición del DESCA.

Son treslas prácticas que se reiteran en los dos proyectos: Horizontalidad y democratización; Poder popular e Intraculturalidad. Acá se puede notar que están relacionadas las apuestas y los referentes para desarrollar los proyectos de extensión universitaria, estableciendo vínculos horizontales con las personas y llevando la universidad al territorio para democratizar la educación.

Frente a las categorías de Senti-saber glolocal del territorio; Saber colectivo; y, Acompañamiento psicosocial, no se desarrollaron ampliamente en el proyecto de Alfabetización, pero los elementos que la componen sí fueron importantes a la hora de desarrollar el proceso.

En el marco de la extensión universitaria crítica se puede establecer una relación de los proyectos de la Universidad Surcolombiana que tienen referentes similares y que da cuenta de la tradición de un modelo acorde a las necesidades de las comunidades de la región donde tienen incidencia la Universidad Surcolombiana.

Dicha tradición se recoge en los elementos teleológicos del Proyecto Educativo Universitario en 2016 que traza una ruta, un horizonte que se venía construyendo en la universidad desde sus inicios con la implementación del proyecto de Alfabetización, y se encuentran vigentes a partir de la implementación del Macroproyecto DESCA.

En esa medida, es importante diseñar una política de extensión universitaria que clarifique, más allá de la distinción entre extensión remunerada o social, una apuesta por desarrollar proyectos de extensión en el marco de la educación comunitaria crítica para acompañar las necesidades y propiciar transformaciones con las comunidades en los territorios donde tiene incidencia la universidad. 
Ahora bien, no sólo es un asunto de extensión, sino que es una discusión que debe dar la Universidad Surcolombiana con las comunidades que habitan en los territorios. Por ende, la movilización social y popular debe marcar la pauta y disputar los escenarios de la universidad para que desde allí se acompañen las transformaciones y mejoren las condiciones.

Esta propuesta de investigación debe leerse en clave de reavivar el debate al interior de la universidad, porque en efecto hay una tradición de este tipo de proyectos que se evidencia desde el año 1984 hasta la actualidad con la continuidad del Macroproyecto DESCA. Por supuesto que hay otras propuestas similares que acá no se pretende desconocer, sino que la invitación está abierta a continuar disputando los escenarios de la universidad.

La disputa debe trascender para problematizar la realidad y problematizar la universidad; frente a las actuales coyunturas políticas, económicas, sociales, culturales, éticas, epistémicas e investigativas, la universidad debe ser referente para propiciar esos debates con la participación de las comunidades que cohabitan territorio con la Universidad Surcolombiana.

Hay que pensar en la democratización de la educación y de los bienes comunales con los que cuenta el territorio, y disputar a la triada universidad, empresa y Estado esos lugares en busca de un modelo de vida para el buen vivir/vivir bien en diálogo permanente con las comunidades y los territorios.

\section{Referencias Bibliográficas}

Acevedo, M., Gómez, R., \& Zúñiga, M. (2016). Pedagogía popular: Una construcción a partir de el diálogo de saberes, la participación comunitaria y el empoderamiento de sujetos sociales. En L. Cendales, M. R. Mejía, \& J. (. Muñoz, Pedagogías y metodologías de la educación popular. "Se hace camino al andar" (pp. 13-32). Bogotá, D.C.: Ediciones Desde Abajo.

Ariztía, T. (2017). La teoría de las prácticas sociales: particularidades, posibilidades y límites. Cinta moebio, 221-234.

Baicué Peña, J. E. (2009). Educación superior en un espacio de frontera. Historia y desarrollo académico de la Universidad Surcolombiana 1970-2010. Tesís Maestría en Historia, Neiva. 
Barragán Girlado, D. F. (Primer Semestre de 2016). Cartografía social pedagógica: entre teoría y metodología. Revista Colombiana de Educación(70), 247-285.

Briones, C. (2020). La horizontalidad como horizonte de trabajo. De la violencia epistémica a la co-labor. En I. Cornejo, \& M. (Rufer, Horizontalidad. Hacia una crítica de la metodología (pp. 59-92). Buenos Aires: CLACSO CONACYT-FONCICYT.

Cabaluz-Ducasse, J. F. (2016). Pedagogías críticas latinoamericanas y filosofía de la liberación: potencialidades de un diálogo teórico-político. Educ.Educ., 19(1), 67-88.

Choachí González, H. A. (2017). Retos de la educación comunitaria y los derechos como alternativa al capital. En S. M. Torres Rincón, Polifonías de la educación comunitaria y popular (pp. 47-90). Bogotá D.C.: Universidad Pedagógica Nacional.

Cierza García, J. A. (2006). Educación comunitaria. Revista de Educación, 765799.

Clavijo Ramírez, A. (2017). La educación comunitaria en el contexto de las propuestas de práctica: reflexiones desde la experiencia con educadores en formación. En S. M. Torres Rincón, Polifonías de la educación comunitaria y popular (pp. 31-46). Bogotá D.C.: Universidad Pedagógica Nacional.

Cuevas Marín, P. (2017). Decolonizar la educación popular - resignficar la comunidad. En S. M. Torres Rincón, Polifonías de la educación comunitaria y popular (pp. 17-30). Bogotá D.C.: Universidad Pedagógica Nacional.

Denzin, N. K., \& Lincoln, Y. (. (2012). Manual de investigación cualitativa. Vol. 1: El campo de la investigación cualitativa. (Vol. I). España: Gedisa.

Freire, P. (1984). ¿Extensión o Comunicación? La concientización en el medio rural. México: Siglo XXI editores, sa.

Galeano Marín, M. E. (2012). Estrategias de investigación social cualitativa. Medellín, Colombia: La Carreta Editores.

Mejía, M. R. (2016). Diálogo-confrontación de saberes y negociación cultural. Ejes de las pedagogías de la educación popular: Una construcción desde el sur. En L. Cendales, M. R. Mejía, \& J. (. Muñoz, Pedagogías y metodologías de la educación popular. "Se hace camino al andar" (pp. 227-249). Bogotá, D.C.: Ediciones Desde Abajo.

Nogueira Beltrão, B. (2020). El conocimiento cuerpo a cuerpo como forma de resistencia ante el racismo(sexismo epistémico. En I. Cornejo, \& M. (. Rufer, Horizontalidad. Hacia una crítica de la metodología (pp. 231-250). Buenos Aires: CLACSO - CONACYT-FONCICYT.

Pérez Luna, E., \& Sánchez Carreño, J. (2005). La educación comunitaria: Una concepción desde la Pedagogía de la Esperanza de Paulo Freire. Revista Venezolana de Ciencias Sociales, 317-329.

Runge Peña, A. K. (s.f.). Analítica empírica de la praxis educativa (escolar y extra-escolar). Aportes al campo disciplinar y profesional de pedagogía desde una teoría de las prácticas. 
Runge Peña, A. K., Gárces Gómez, J. F., \& Muñoz Gaviria, D. A. (s.f.). La Pedagogía como Campo Profesional y Disciplinar.

Torres Carrillo, A. (2013). El retorno a la comunidad. Problemas, debates y desafios de vivir juntos. Bogotá, D.C.: CINDE - EL BÚHO.

Valdés Gutiérrez, G. (2018). Reflexiones ético-políticas desde los talleres de paradigmas emancipatorios. En X. J. Leyva Solano, Prácticas otras de conocimiento(s). Entre crisis, entre guerras (pp. 321-348). México: Cooperativa Editorial RETOS, Taller Editorial La Casa del Mago, CLACSO, 3 tomos.

Valladares, L. (28 de junio de 2017). La "práctica educativa" y su relevancia como unidad de análisis ontológico, epistemológico y sociohistórico en el campo de la educación y la pedagogía. Perfiles Educativos, XXXIX(158), 186-203.

Vasilachis de Gialdino, I. ((2006). Estrategias de investigación cualitativa. Barcelona: Gedisa. 